Derecho y Realidad

Vol.14 Núm. 27 • Enero Junio de 2016

Págs. 137-149·ISSN:1692-3936

\title{
Vulneración de los derechos humanos en la educación tunjana
}

\author{
Violation of human rights in Tunja education
}

\author{
Manuel Ricardo Gómez Barreto*
}

\section{Resumen}

La educación colombiana asume politicas de calidad y gratuidad encaminadas a proteger los derechos humanos en el marco pedagógico. A continuación se aborda un análisis del desarrollo de estas politicas en la ciudad de Tunja, para dar una visión respecto a la vulneración de los derechos humanos en la enseñanza, asumiendo los diferentes acuerdos realizados para su protección, sin desligar los diferentes procesos que una educación de calidad requiere. Se tuvieron en cuenta las políticas internacionales de protección y calidad educativa, y el análisis sustenta esta información con estadísticas reales, emitidas por las autoridades del municipio.

Estas estadísticas son empleadas para establecer la pertinencia y eficiencia del sistema actual frente a las posibles violaciones a los derechos humanos -que se presentan en el afán de ampliar cobertura, sin tener en cuenta otros aspectos contextuales, relacionados con la eficacia-, así como para analizar la oferta integral de calidad en la ciudad.

Fecha de aceptación: 15 de septiembre de 2015

Concepto de recepción: 18 de septiembre de 2015

Fecha de aprobación: 29 de septiembre de 2015

* Licenciado en Ciencias Sociales, docente de Ciencias Sociales y Filosofía en Institución Educativa Técnica Rancho Grande del municipio de Rondón, departamento de Boyacá. Magistrando en Derechos Humanos, UPTC Tunja (Boyacá). manuelricardo.gomez@uptc.edu.co 


\section{Palabras clave}

Calidad, derechos humanos, educación, gratuidad, violación.

\section{Abstract}

Colombian education assumes quality and gratuity policies aimed at protecting human rights in pedagogical framework. This paper addresses an analysis of the development of these policies in the city of Tunja, to give an overview about the violation of human rights in teaching, assuming the various agreements made for their protection, without separating the different processes that quality education requires. It was taken into account international policies of educational protection and quality, and the analysis is based on actual statistics issued by the local authorities.

These statistics are used to establish the relevance and efficiency of the current system against possible human rights violations -which are presented in the effort to expand coverage without considering other contextual aspects related to effectiveness-, as well as to analyze the comprehensive offer in the city.

\section{Keywords}

Quality, human rights, education, gratitousness, rape. 


\section{Introducción}

La educación colombiana es asumida actualmente con una política que aborda tópicos relacionados con calidad y gratuidad, ratificada en el período 2010-2014. Dicha gratuidad busca "...educar con pertinencia para la innovación y la prosperidad", tiende al fortalecimiento de la gestión educativa, para hacer de la educación un modelo de gestión y administración pública, como ejemplo de buen gobierno. Sin embargo, en el afán de obtener una mayor cobertura e inclusión educativa, extendida a “...1.'706.807 niños menores de cinco años que no tienen acceso a una oferta integral de calidad... y recuperar de esta manera el $53 \%$ de la población escolar rural que ha desertado y el $16 \%$ de la población escolar de la zona urbana", según lo indican las estadísticas del Dane y las encuestas del Sisbén a noviembre de 2009, pareciera que se está limitando la calidad por ampliar la cobertura.

La política del Gobierno está fundamentada en que la educación de calidad es la que propende la formación de mejores seres humanos, éticos y respetuosos de los derechos humanos; sin embargo, los países de la región Caribe han realizado esfuerzos considerables para aumentar los años de la educación obligatoria, incrementar la cobertura, mejorar la infraestructura, diseñar nuevos currículos y formar a los docentes, entre otros aspectos.

No obstante, en Colombia para el 2012, según las estadísticas del Ministerio de Educación Nacional (MEN, 2012), se aumentó la cobertura en todos los niveles educativos en 1.3 millones para un total de 11.3 millones de estudiantes; además, se invirtió en construcción y mejoramiento de infraestructura de 12732 aulas con 1.2 billones, y como lo indican las estadísticas del MEN, se incrementó la conectividad y acceso de las nuevas tecnologías, pues pasó a un $87 \%$, es decir, a un computador por cada 21 estudiantes en promedio. Lo cual es considerablemente bajo para poder hablar de calidad y eficiencia, por ello es necesario preguntarse si con estas estadísticas se vulneran los derechos humanos en la educación tunjana respecto a la calidad y la gratuidad pedagógica.

La Declaración de los Derechos Humanos establece en sus Artículos 12, 26 y 27 la protección de la dignidad humana, la reputación, el derecho a la educación, la cual debe ser gratuita y obligatoria, la cual propende al pleno desarrollo de la personalidad humana y el respeto por los derechos humanos, tomando parte de manera activa en los avances culturales de la comunidad.

Para verificar esta situación se hace necesario la recopilación y análisis de la información de las estadísticas locales y nacionales emitidas por las autoridades municipales, el MEN (Ministerio de Educación Nacional) y el Dane (Departamento 
Nacional de Estadísticas), entre otras, indispensables para poder analizar la protección de los derechos humanos en la educación tunjana, teniendo en cuenta la gratuidad y la ampliación en la cobertura por parte del municipio y atendiendo la política nacional.

\section{Vulneración de los derechos humanos en la educación tunjana respecto a la calidad y la gratuidad pedagógica}

La calidad y la gratuidad en la educación colombiana, ofrecida por las políticas gubernamentales, quiere mostrar un plan de acción por parte del Gobierno tendiente a buscar el cumplimiento de los derechos humanos, sin detenerse a analizar qué pasa con ese $11 \%$ de los estudiantes que no asisten a las aulas de clase. A la vez, este estudio pretende verificar si hablar de cobertura y calidad implica de forma directa o no, dar cumplimiento a la protección de los derechos humanos en Colombia, propendiendo a una educación que forme en valores, que forme por la paz y para la paz.

Las políticas orientadas a la consecución de recursos indican la protección de estos derechos, no solo para los estudiantes, sino para el docente, dentro de un mismo marco contextual que brinde la oportunidad de adentrarnos en la búsqueda de información, que verifique si se cumplen o no los derechos en el país, sin desvincular el sistema educativo, el cual borra las fronteras de la discriminación en todo sentido frente a la población más vulnerable. Esta investigación atraviesa por etapas de recolección y síntesis de informaciones censales y de estudios adelantados que corroboran o no la protección de los derechos humanos en la escuela colombiana, sectorizando las estadísticas brindadas por el MEN y constatando el cumplimiento de políticas para la prosperidad, con el fin de ser más objetivo en las posibles conclusiones que se obtengan a lo largo de la investigación.

Los derechos humanos establecen la protección y garantía de la educación de calidad, la cual debe ser ofrecida de manera estándar, sin necesidad de discriminación alguna, pero ¿se están protegiendo realmente los derechos humanos en la educación tunjana, respecto a la calidad y gratuidad pedagógica? Establecer esta realidad es una tarea que requiere recolección de información y análisis de la misma, para establecer conclusiones que evidencien los diferentes procesos de garantía ofrecidos por las autoridades locales, mediante una investigación de tipo descriptivo que argumente la defensa o no de los derechos humanos de los niños en la ciudad de Tunja.

Corroborando las estadísticas a 2010, se puede verificar que 11.122.874 estudiantes se encuentran matriculados actualmente en el país entre sectores oficiales y no oficiales (citar la fuente, año y pág. consultada) lo que quiere decir que existe 
una cobertura neta del $89 \%$; indica igualmente, un alto porcentaje en la calidad educativa, fusionando instituciones, aumentando cobertura, pero manteniendo el mismo número de docentes, lo cual nos deja entrever que se pretende mejorar calidad disminuyendo recursos.

Al disminuir los recursos y aumentar la cobertura surge un interrogante entre lo que busca el Gobierno nacional y lo que realmente se está creando en el contexto escolar y el número de estudiantes por profesor.

En cuanto a la situación de los derechos humanos en Colombia, según se indica en la declaración de Amnistía Internacional ante la Decimonovena Sesión del Consejo de Derechos Humanos de la ONU del 27 de febrero de 2012, es muy pobre el compromiso del Gobierno colombiano en la prevención de la violencia contra niños y mujeres, y a la vez recomienda al Gobierno perseguir con más ímpetu la realización de su compromiso expreso con los derechos humanos y ponerle fin a la impunidad. Otras recomendaciones al Gobierno colombiano son:

a. Desvincular a la escuela del conflicto y definirla y protegerla como "espacio de paz".

b. Aclarar la legitimidad de los derechos humanos y desarrollar su enseñanza y aprendizaje con plena participación de los defensores de derechos humanos, del personal docente y de los alumnos y alumnas para adaptar el proceso educativo al contexto colombiano.

c. Desarrollar mecanismos que hagan efectiva la eliminación de toda discriminación de las niñas embarazadas y niñas - madres como lo postuló la Corte Constitucional.

Lo cual reafirma los estudios realizados por la Revista Latinoamericana de Educación (2010) que recomienda:

Generar un espacio de intercambio, debate y reflexión con relación a los desafíos que supone avanzar hacia una educación inclusiva para todos, en el ámbito de las políticas, la cultura y las prácticas educativas.

Difundir estudios, investigaciones, programas y experiencias innovadoras, que aporten al conocimiento y desarrollo de escuelas que acojan y respondan a la diversidad de necesidades educativas de los estudiantes.

Promover propuestas y estrategias que apunten a la mejora de la calidad y equidad de los sistemas educativos, con especial atención en los sectores y grupos más vulnerables. 
Lo anterior deja entrever la lucha por una educación gratuita de calidad, pero vale la pena preguntar si se aplica el cumplimiento de referentes legales, para promover el respeto de los derechos humanos, la educación y conocimiento de los mismos por parte de la comunidad educativa.

“(...) Para las Naciones Unidas la educación es un derecho humano fundamental y uno de los instrumentos más eficaces para promover los derechos humanos," teniendo en cuenta políticas internacionales de calidad que solo buscan la dignificación de la vida de las personas, y en especial, la protección de los niños y su educación, con el fin de lograr un desarrollo integral del hombre, promoviendo igualdad y eficacia, que responda no solo a las metas del sector y a los objetivos de un gobierno que propenda la protección del individuo.

La calidad de la educación, en cuanto derecho fundamental de todas las personas, ha de reunir, desde la perspectiva de la OREALC/UNESCO (Oficina Regional de Educación para América Latina), las siguientes dimensiones: respeto de los derechos, relevancia, pertinencia, equidad y eficiencia y eficacia. Dando un valor significativo a los distintos procesos educativos, los cuales deben asegurar las diferentes dimensiones de una política de calidad de una manera significativa, que no entorpezca un verdadero desarrollo de la zona, sino, por el contrario, brinde garantías de cumplimiento.

En la actualidad, Colombia es reconocida internacionalmente por sus políticas de prosperidad para todos, dando un vuelco total a lo que es, en realidad, la calidad educativa, pero ¿es verdad tanta maravilla?

Para analizar si las políticas actuales abordan de manera adecuada la problemática de la cobertura y la gratuidad con estándares de calidad, basta dar un vistazo a la sociedad y, de manera lógica, se deben examinar diferentes contextos donde el fenómeno por estudiar arroje resultados que permitan vislumbrar la realidad de un municipio frente a las presiones de una política local, departamental y nacional.

Son muchos los frentes a los que debe responder la Secretaría de Educación de cualquier municipio certificado, sin embargo, para verificar que se dé cumplimiento a lo establecido por las Naciones Unidas, se escoge la ciudad de Tunja, ubicada en el departamento de Boyacá, con el fin de corroborar evidencias locales de los entes controladores, de todos los tipos de violencia que contra los menores educandos o en edad escolar se hallan presentado en todos los niveles, verificando a la vez, las acciones tomadas por las diferentes autoridades locales que facilitan los avances en materia de protección social a los tunjanos y a los niños, niñas, jóvenes y adolescentes en edad escolar. 
Si bien es cierto los derechos humanos abarcan políticas claras en materia de defensa, también es claro que la educación debe ser de calidad, pero para ello se deben tener en cuenta otros tópicos que rodean dicha calidad.

Las políticas nacionales orientan actualmente las diferentes evaluaciones por competencias, las cuales, según el MEN, son aquellas que permiten medir el grado de calidad que se brinda en las diferentes instituciones educativas, cayendo, como lo indica EPT/PRELAC (Educación para todos/ Proyecto Regional de Educación para América Latina y el Caribe), en sesgar la formulación de políticas como la actividad de un docente y la realidad escolar. Dentro de los sesgos más relevantes se encuentran:

El reduccionismo instrumental: entendido como la limitación de aspectos mensurables, controlando únicamente los resultados de pruebas externas, dejando de lado particularidades que difícilmente pueden evaluarse, instrumentos entendidos por la EPT/PRELAC, tales como "la creatividad, la resolución de conflictos o el cuidado del medio ambiente, entre otras."

Este aspecto en Colombia es visible en el quehacer de los educadores y educandos, por cuanto la preparación integral que buscan los docentes se ve limitada por las evaluaciones realizadas por el Icfes, que desatienden la verdadera formación de la persona y aspectos subjetivos, y tienden a hacer creer que si un estudiante obtiene puntajes altos en algunas áreas del conocimiento en las pruebas saber, la calidad educativa es de lo mejor en dicha institución, sin detenerse a analizar los aspectos contextuales de las diferentes zonas del país en cuanto a infraestructura, conectividad, recursos, talento humano, zonas de difícil acceso, factores económicos, entre otros.

De forma particular, cabe analizar ¿por qué la educación pública para el país excluye de la educación superior a aquellos estudiantes que no obtienen buenos resultados en las Pruebas Saber?

Para el caso particular, la ciudad de Tunja obtiene la posición 16 con el colegio Los Ángeles, según la clasificación de Leodoncel para el 2012, sin embargo, dicha posición no aplica para los colegios oficiales de la ciudad. Es necesario aclarar que el colegio Los Ángeles es de carácter privado, que su población escolar es mucho menor que la de los colegios oficiales, que los niños que estudian en esta institución son de estrato cuatro alto, mientras que los niños de los colegios oficiales son de estrato uno en su gran mayoría. Alrededor de estos parámetros se vislumbra que a pesar de ampliar la cobertura a nivel local, los colegios en sus pruebas externas no evidencian una relación directa de calidad y eficiencia frente a las pruebas aplicadas. Lo cual debería ser una señal para el Gobierno nacional, pues queda claro que los 
colegios privados cuentan con recursos que no llegan a los estratos bajos, pese al intento de mantener procesos formativos con los estándares emitidos por el MEN y brindar con ello igualdad en educación a todos los niños del país. Y lo que es peor, se asumen políticas represivas en contra de los colegios que no muestren buenos resultados en las pruebas Saber, manipulando de esta manera la política educativa, forzando el sistema educativo sin asumir la realidad que el sector enfrenta en la actualidad.

2. La normatividad engañosa: donde se termina valorando solo aquello que es objeto de evaluación, dejando de lado la preocupación de formar personas, que respeten, que aporten de manera oportuna a la sociedad, que sean capaces de tomar decisiones frente a las diferentes situaciones que se les presenten. Dicha normatividad deja entrever dentro de la política de "prosperidad para todos" como el aumento de la cobertura a nivel nacional supuestamente vela por la protección de los derechos humanos, pero, analicemos entonces, ¿qué pasa con la relación docente-estudiante?

Para el MEN es un reto hablar de calidad educativa y establece dentro de su plan de acción ampliar la cobertura y la gratuidad sin aumentar el gasto educativo y disminuyendo el número de docentes en las instituciones educativas oficiales.

Actualmente, Colombia invierte en educación aproximadamente el $7.3 \%$ del PIB en el sector educativo, mientras que países como Cuba invierten el $13.5 \%$ del PIB, Estados Unidos el 7.7 \%, República de Corea el 7.6 \%, entre otros, lo que indica que gastan más en educación. Comparando con Finlandia, esta aparentemente invierte solo el $5.8 \%$ del PIB en educación, y Colombia estaría por encima de su inversión $1.5 \%$ del PIB en inversión del gasto educativo. Esto indicaría que Colombia está dentro de los límites normales de inversión en gasto educativo, pero, si bien es cierto que Finlandia ofrece una calidad educativa excelente, la cual entendida desde este punto de vista haría ver a Colombia como un país adelantado. Es necesario aclarar que "Finlandia cuenta con una población de 5'.375.276" habitantes aproximadamente, según el Banco Mundial, mientras Colombia tiene 47'.121.089 habitantes aproximadamente, y haciendo una relación directa de inversión, Colombia debería invertir como mínimo el 50.84 \% del PIB para alcanzar los logros obtenidos por Finlandia. Desde este punto de vista, analizar la calidad para un gasto tan pobre deja entrever la verdadera realidad que azota al país.

Ahora, en Tunja la población escolar, teniendo en cuenta los sectores oficiales y no oficiales, permite percibir como, a pesar de aumentar la matrícula de estudiantes a 39604 según las estadísticas del MEN, el número de docentes disminuye en Boyacá pasando de 11225 a 10891 . Es aquí donde el punto crítico comienza a mostrar su verdadera faceta de cumplimiento hacia normatividad engañosa, frente a acuerdos 
internacionales de calidad donde se beneficie a los estudiantes y no se ligue al sistema educativo a cifras engañosas que quieren hacer ver el sector como una fortaleza del país cuando la realidad es otra.

3. "Reduccionismo racionalista (...) que consiste en confundir los fenómenos con las explicaciones de los mismos. El fenómeno del aprendizaje se produce a través de procesos de interacción entre el docente y los alumnos y de éstos entre sí, y lo que cada uno aporta".

En Tunja, dicha realidad debe reconocer que ampliar cobertura sin ampliar número de docentes hace del sistema educativo una empresa dedicada a disminuir inversión pero a exigir materia prima de excelente calidad, para sostener un sistema fracasado. Son muchos los esfuerzos que en el ámbito de la docencia se deben unir en pro de la educación de los jóvenes, y los docentes pasan de ser orientadores y facilitadores dentro de un proceso de formación a ser máquinas de trabajo que deben registrar papelería, perdiendo tiempo valioso de su esencia escolar, motivando Decretos como el 1278, que presionan al docente con una serie de políticas que incluso violan el derecho a la igualdad de quienes preocupados por sus estudiantes pretenden trabajar sin herramientas en pro de una educación enfocada a exámenes nacionales que no miden objetivamente el aprendizaje de un niño.

Obviamente, la labor de un docente requiere entrega y sacrificio, entendido desde un punto de vista epistemológico por propender y velar a como dé lugar de su estudiante, pasando a ser secretario, contador, sicólogo, doctor, amigo y en ocasiones hasta padres de niños ajenos, situación que sucede a menudo en las instituciones educativas.

Cuando el sistema falla, comienza a aparecer una serie de violaciones a la protección de los derechos humanos de los niños, niñas, jóvenes y adolescentes en edad escolar, los cuales se ven reflejados en deserción escolar, denuncias por parte de los agredidos o personas cercanas a estos. Situación que aclara que no por el hecho de brindar gratuidad educativa y ampliar la cobertura se están respetando los derechos humanos de los niños, y es precisamente lo que se desea analizar, teniendo en cuenta las estadísticas recopiladas.

El municipio de Tunja cuenta actualmente con 13 instituciones educativas de carácter oficial, con 36 sedes fusionadas y 101 establecimientos educativos de carácter privado. A partir de esta aclaración, se analizan los índices de deserción escolar, tomando como referencia el cálculo deserción interanual 2010 a 2012 del municipio de Tunja sector oficial, realizado por Carolina Torres Tovar, líder de cobertura de la Secretaría de Educación Municipal, donde se puede apreciar que existe aumento de 
cobertura y gratuidad de la educación básica. Sin embargo, si bien existe aumento de población con edad escolar en las instituciones educativas del municipio, aumenta también la deserción escolar, pasando de 597 estudiantes en el 2011 a 653 en el 2012, estudiantes que asumen la deserción debido a causas tales como:

- Condiciones de la zona de ubicación de los establecimientos educativos.

- Condiciones de los establecimientos educativos.

- Condiciones de los hogares.

- Condiciones individuales de los estudiantes de Tunja.

Las cuales tienen su justificación en la manera como algunos padres de familia perciben la seguridad de sus hijos; por ejemplo, la ola invernal que azotó a la ciudad el año anterior, la lejanía de las instituciones educativas en relación con el lugar de residencia de los niños, el matoneo escolar, los conflictos y violencia generada entre compañeros de clase, la falta de recursos económicos de las familias para poder continuar con el apoyo a sus hijos menores con edad de escolarización, la falta de infraestructura adecuada de algunas instituciones, los conflictos familiares, obligaciones con el hogar o familiares que están a cargo de los niños, las dificultades académicas y en ocasiones algunos estudiantes definitivamente aclaran que simplemente no les gusta el estudio.

Según el Instituto Colombiano de Bienestar Familiar ICBF, en las oficinas del equipo de la Defensoría de Familia se ha presentado una serie de denuncias de abuso o han sido remitidas por alguna autoridad competente las condiciones de abuso en contra de menores en cuanto a:

\section{Formación.}

2. Libertad.

3. Integridad sexual.

4. Violencia sexual, entre otros.

De los datos obtenidos y analizados por el ICBF se puede entender que "el delito que más prevalece son los actos sexuales con menor de 14 años, con un $45 \%$; como tocamientos, caricias, masturbación y exhibicionismo; seguido por un incremento en el delito de acceso carnal abusivo, reflejado en un $32 \%$, lo que implica la penetración de cualquier parte del cuerpo del agresor u objeto en el cuerpo del niño, niña o adolescente".

Es importante aclarar que las familias usuarias de Caivas (Centro de Atención Integral a las Víctimas de Abuso Sexual) refieren sentimientos ambivalentes cuando se presenta la tipificación de delitos sexuales, por cuanto se tranquilizan al observar 
que el dictamen de medicina legal evidencia la ausencia de penetración y por otro lado, existe la creencia de que al no haber lesiones evidentes en el área genital, el proceso legal pueda ser poco creíble en la etapa probatoria.

En las oficinas de Caivas tan solo 92 casos son denunciados o remitidos a la misma y en algunos casos son de niños que desertaron del sistema escolar, y es allí donde surge otro interrogante, ¿se protegen los derechos humanos de los niños en la educación?

Observar un panorama como este, indica una real violación de los derechos humanos en cuanto a educación se refiere, por cuanto las políticas internacionales pretenden lograr una educación precisa y pertinente que cumpla con requisitos que dependen de disponibilidad, accesibilidad, no discriminación, accesibilidad material y económica, aceptabilidad y adaptabilidad, pero los niños de la ciudad se ven inmersos en un sistema que para nada orienta una opción clara de ser eficientes y eficaces. La ciudad debe atravesar por un ente de control estricto que dirija sus recursos a la educación con el propósito de finalizar la desigualdad social, económica y cultural, y para ello debe realizar un análisis exhaustivo del deber que se tiene con la sociedad para formar personas que le aporten a esta en forma pertinente, sin distinción alguna y con igualdad de oportunidades.

\section{Conclusiones}

1. La educación es un derecho fundamental; no obstante, lo importante de esta premisa es definir que sea de calidad y gratuita, al menos en sus primeras instancias, situación que en el país no se lleva a cabo por procesos gubernamentales que ven la educación como un negocio económico y no como un derecho fundamental. La calidad educativa no tiene que ver con procesos proteccionistas y paternalistas por parte del Estado a las familias más vulnerables, sino que tiene que ser enfocada a buscar estrategias encaminadas a la formación de los estudiantes en procesos investigativos que requieren inversión de capital en cuestión de docentes, infraestructura de las instituciones, cursos con menos estudiantes, accesibilidad a procesos integradores como las TICS, y no a procesos metodológicos inoficiosos, como el diligenciamiento de papelería en muchas ocasiones inútil, que hace que se pierda tiempo valioso en lo realmente importante que es la formación de los estudiantes. Establecer procesos de igualdad entre los docentes, pues al existir dos decretos que los rigen, hace que no se trabaje en forma mancomunada e imparcial; dicho en otras palabras, a los docentes del Decreto 1278 con relación a los del 2277 se les hacen menos exigencias, además por pertenecer a decretos distintos existe algo que coacciona la igualdad y que solo atrasa el proceso educativo que se ha vuelto tan dinámico en la última década. 
2. La educación debe tener mayor atención en cuestión de inversión, pues al analizar los recursos que se utilizan para esta se puede ver que son pocos en relación con otros campos, como por ejemplo, la guerra, sin darse cuenta que el verdadero sentido de desarrollo de una nación se ve reflejado en su educación. Es por esto que el país vive una constante guerra, pues si se analiza el grado de escolaridad de los actores del conflicto armado colombiano, la mayoría solo alcanza la básica primaria. ¿Por qué en vez de gastar tanto dinero en armamento y guerra no se invierte en procesos educativos de sensibilización? La educación es la mejor estrategia para acabar la ignorancia, no vista como el hecho de no saber leer y escribir, sino entendida como tener la capacidad de ser competente laboralmente, siendo incluido en programas de formación que ofrece el Sena, entidad que se ve involucrada en los procesos educativos actuales, brindándoles la oportunidad a los estudiantes que no pueden acceder a la educación superior a formarse en el campo técnico desde su bachillerato.

\section{Referencias}

Calixto, S. V. (2012). Diagnóstico de la violencia sexual de niños, niñas y adolescentes del municipio de Tunja - 2012. Tunja, Colombia: Instituto Colombiano de Bienestar Familiar, regional Boyacá, Unidad Especializada de Delitos Sexuales - Caivas Tunja.

Cerda, H. \& Bunge, M. (1998). Los elementos de la investigación. (1ª reimp.). Bogotá: El Búho.

Ministerio de Educación de Colombia (2002). Decreto Ley 1278 de 2002 Estatuto de Profesionalización Docente. Bogotá: Imprenta Nacional.

Ministerio de Educación de Colombia. (2010, 10 de nov.). Educación de calidad, el camino para la prosperidad, pacto nacional por el mejoramiento de la calidad educativa. Bogotá: Imprenta Nacional.

Ministerio de Educación de Colombia (2010). Plan Nacional de Derechos Humanos Planedh 2010-2014. Bogotá: Imprenta Nacional.

Organización de las Naciones Unidas -ONU- (2000). ABC de las Naciones Unidas. Nueva York: ONU, Departamento de Información Pública.

Revista Latinoamericana de Educación Inclusiva (2010). Escuela de Educación diferencial. Revista Latinoamericana de Educación Inclusiva, 4(1).

Unesco (2007). Educación de calidad para todos, un asunto de derechos humanos. Documento de discusión sobre políticas educativas. En II Reunión Gubernamental del Proyecto Regional de Educación para América Latina y el Caribe (EPT/ PRELAC), celebrada en Buenos Aires, Argentina durante el 29, 30 de marzo de 2007. 